\title{
ON RECURRENCE FORMULAE
}

\author{
by T. M. MACROBERT
}

(Received 20th October, 1955)

1. Introductory. The recurrence formulae for the Bessel, Legendre, hypergeometric and other such functions can all be related to each other by means of the $E$-functions. In this paper it will be shown how, starting from known recurrence formulae for the hypergeometric function, others can be derived. The $E$-function formulae are deduced in $\S 2$, and the others in $\S 3$.

2. E-function formulae. Many recurrence formulae are known ([1], [2]) for the hypergeometric function. From these the following four have been selected for consideration.

$$
\begin{gathered}
\gamma F(\alpha, \beta-1 ; \gamma ; z)-\gamma F(\alpha-1, \beta ; \gamma ; z)+(\alpha-\beta) z F(\alpha, \beta ; \gamma+1 ; z)=0 . \\
\gamma(\alpha-\beta) F(\alpha, \beta ; \gamma ; z)-\alpha(\gamma-\beta) F(\alpha+1, \beta ; \gamma+1 ; z)+\beta(\gamma-\alpha) F(\alpha, \beta+1 ; \gamma+1 ; z)=0 . \\
\gamma(\gamma+1) F(\alpha, \beta ; \gamma ; z)-\gamma(\gamma+1) F(\alpha, \beta ; \gamma+1 ; z)-\alpha \beta z F(\alpha+1, \beta+1 ; \gamma+2 ; z)=0 . \\
(\beta-\alpha) F(\alpha, \beta ; \gamma ; z)+\alpha F(\alpha+1, \beta ; \gamma ; z)-\beta F(\alpha, \beta+1 ; \gamma ; z)=0 .
\end{gathered}
$$

Now in (1) replace $\alpha, \beta, \gamma$ and $z$ by $\alpha_{1}, \alpha_{2}, \rho_{1}-1$ and $-1 / z$, and multiply by $\Gamma\left(\alpha_{1}\right), \Gamma\left(\alpha_{2}\right)$ and $1 / \Gamma\left(\rho_{1}\right)$; then the formula can be written

$$
\left(\alpha_{1}-\alpha_{2}\right) E\left(\alpha_{1}, \alpha_{2}: \rho_{1}: z\right)=\left(\alpha_{2}-1\right) z E\left(\alpha_{1}, \alpha_{2}-1: \rho_{1}-1: z\right)-\left(\alpha_{1}-1\right) z E\left(\alpha_{1}-1, \alpha_{2}: \rho_{1}-1: z\right) ;
$$

and, when this is generalised, it becomes

$$
\begin{aligned}
&\left(\alpha_{1}-\alpha_{2}\right) z^{-1} E\left(p ; \alpha_{r}: q ; \rho_{s}: z\right)=\left(\alpha_{2}-1\right) E\left(\alpha_{1}, \alpha_{2}-1, \ldots, \alpha_{p}-1: q ; \rho_{s}-1: z\right) \\
&-\left(\alpha_{1}-1\right) E\left(\alpha_{1}-1, \alpha_{2}, \alpha_{3}-1, \ldots, \alpha_{p}-1: q ; \rho_{s}-1: z\right) .
\end{aligned}
$$

In the same manner, from formulae (2) to (4) the following can be derived.

$$
\begin{aligned}
& \left(\alpha_{1}-\alpha_{2}\right) E\left(p ; \alpha_{r}: q ; \rho_{s}: z\right)=\left(\rho_{1}-\alpha_{2}\right) E\left(\alpha_{1}+1, \alpha_{2}, \ldots, \alpha_{p}: \rho_{1}+1, \rho_{2}, \ldots, \rho_{q}: z\right) \\
& -\left(\rho_{1}-\alpha_{1}\right) E\left(\alpha_{1}, \alpha_{2}+1, \alpha_{3}, \ldots, \alpha_{p}: \rho_{1}+1, \rho_{2}, \ldots, \rho_{a}: z\right) \text {. } \\
& E\left(p ; \alpha_{r}: q ; \rho_{s}: z\right)=\rho_{1} E\left(p ; \alpha_{r}: \rho_{1}+1, \rho_{2}, \ldots, \rho_{q}: z\right) \\
& -z^{-1} E\left(p ; \alpha_{r}+1: \rho_{1}+2, \rho_{2}+1, \ldots, \rho_{q}+1: z\right) \text {. } \\
& \left(\alpha_{1}-\alpha_{2}\right) E\left(p ; \alpha_{r}: q ; \rho_{s}: z\right)=E\left(\alpha_{1}+1, \alpha_{2}, \ldots, \alpha_{p}: q ; \rho_{s}: z\right) \\
& -E\left(\alpha_{1}, \alpha_{2}+1, \alpha_{3}, \ldots, \alpha_{p}: q ; \rho_{s}: z\right)
\end{aligned}
$$

From these formulae others can be deduced. For instance, on applying (5) to the terms on the right of $(5)$, it is found that

$$
\begin{aligned}
& \left(\alpha_{1}-\alpha_{2}-1\right)\left(\alpha_{1}-\alpha_{2}+1\right)\left(\alpha_{1}-\alpha_{2}\right) E\left(p ; \alpha_{r}: q ; \rho_{8}: z\right) \\
& =\left(\alpha_{1}-\alpha_{2}-1\right)\left(\alpha_{2}-1\right)\left(\alpha_{2}-2\right) z^{2} E\left(\alpha_{1}, \alpha_{2}-2, \ldots, \alpha_{p}-2: q ; \rho_{s}-2: z\right) \\
& -2\left(\alpha_{1}-\alpha_{2}\right)\left(\alpha_{1}-1\right)\left(\alpha_{2}-1\right) z^{2} E\left(\alpha_{1}-1, \alpha_{2}-1, \alpha_{3}-2, \ldots, \alpha_{p}-2: q ; \rho_{s}-2: z\right) \\
& +\left(\alpha_{1}-\alpha_{2}+1\right)\left(\alpha_{1}-1\right)\left(\alpha_{1}-2\right) z^{2} E\left(\alpha_{1}-2, \alpha_{2}, \alpha_{3}-2, \ldots, \alpha_{p}-2: q ; \rho_{s}-2: z\right) \text {. }
\end{aligned}
$$

Again, on applying (5) to the terms on the right of (8), we get

$$
\begin{aligned}
\left(\alpha_{1}-\alpha_{2}-1\right) & \left(\alpha_{1}-\alpha_{2}+1\right)\left(\alpha_{1}-\alpha_{2}\right) E\left(p ; \alpha_{r}: q ; \rho_{s}: z\right) \\
= & \left(\alpha_{1}-\alpha_{2}-1\right)\left(\alpha_{2}-1\right) z E\left(\alpha_{1}+1, \alpha_{2}-1, \ldots, \alpha_{p}-1: q ; \rho_{s}-1: z\right) \\
& -\left(\alpha_{1}+\alpha_{2}-1\right)\left(\alpha_{1}-\alpha_{2}\right) z E\left(\alpha_{1}, \alpha_{2}, \alpha_{3}-1, \ldots, \alpha_{p}-1: q ; \rho_{s}-1: z\right) \\
& +\left(\alpha_{1}-\alpha_{2}+1\right)\left(\alpha_{1}-1\right) z E\left(\alpha_{1}-1, \alpha_{2}+1, \alpha_{3}-1, \ldots, \alpha_{p}-1: q ; \rho_{s}-1: z\right) .
\end{aligned}
$$


Finally, apply (5) to the terms on the right of (6), and so obtain

$$
\begin{aligned}
& \left(\alpha_{1}-\alpha_{2}-1\right)\left(\alpha_{1}-\alpha_{2}+1\right)\left(\alpha_{1}-\alpha_{2}\right) E\left(p ; \alpha_{\tau}: q ; \rho_{s}: z\right) \\
& \quad=\left(\alpha_{1}-\alpha_{2}-1\right)\left(\rho_{1}-\alpha_{2}\right)\left(\alpha_{2}-1\right) z E\left(\alpha_{1}+1, \alpha_{2}-1, \ldots, \alpha_{p}-1: \rho_{1}, \rho_{2}-1, \ldots, \rho_{q}-1: z\right) \\
& \quad-\left\{\rho_{1}\left(\alpha_{1}+\alpha_{2}-1\right)-2 \alpha_{1} \alpha_{2}\right\} z E\left(\alpha_{1}, \alpha_{2}, \alpha_{3}-1, \ldots, \alpha_{p}-1: \rho_{1}, \rho_{2}-1, \ldots, \rho_{q}-1: z\right) \\
& \quad+\left(\alpha_{1}-\alpha_{2}+1\right)\left(\rho_{1}-\alpha_{1}\right)\left(\alpha_{1}-1\right) z E\left(\alpha_{1}-1, \alpha_{2}+1, \alpha_{3}-1, \ldots, \alpha_{p}-1: \rho_{1}, \rho_{2}-1, \ldots, \rho_{q}-1: z\right) . .
\end{aligned}
$$

The formula for the derivative is

$$
\frac{d}{d z} E\left(p ; \alpha_{r}: q ; \rho_{s}: z\right)=\frac{1}{z^{2}} E\left(p ; \alpha_{r}+1: q ; \rho_{s}+1: z\right) .
$$

3. Special Cases. The recurrence formulae for the Bessel and Legendre functions are special cases of the formulae of $\S 2$.

Bessel functions. In (7) take $p=0, q=1, \rho_{1}=n$ and replace $z$ by $4 / z^{2}$; then, since

$$
\begin{array}{r}
J_{n}(z)=\left(\frac{1}{2} z\right)^{n} E\left(: n+1: 4 / z^{2}\right), \\
J_{n-1}(z)-2 n z^{-1} J_{n}(z)+J_{n+1}(z)=0 .
\end{array}
$$

Next, in (10) put $p=2, q=0, \alpha_{1}=\frac{1}{2}+n, \alpha_{2}=\frac{1}{2}-n$, and replace $z$ by $2 z$; then, since

$$
\begin{gathered}
E\left(\frac{1}{2}+n, \frac{1}{2}-n:: 2 z\right)=\sec n \pi \sqrt{ }(2 \pi z) e^{z} K_{n}(z), \\
2 n z^{-1} K_{n}(z)=K_{n+1}(z)-K_{n-1}(z) . \quad \ldots \ldots . .
\end{gathered}
$$

Legendre functions. In (10) put $p=2, q=1, \alpha_{1}=-n, \alpha_{2}=n+1, \rho_{1}=m+2$, and replace $z$ by $2 /(z-1)$; then, since

$$
\begin{array}{r}
T_{n}^{-m}(z)=-\pi^{-1} \sin n \pi\left(\frac{1-z}{1+z}\right)^{1 m} E\left(-n, n+1: m+1: \frac{2}{z-1}\right) \\
(2 n+1) \sqrt{ }\left(1-z^{2}\right) T_{n}^{-m-1}(z)=T_{n-1}^{-m}(z)-T_{n+1}^{-m}(z) .
\end{array}
$$

Again, in (11) put $p=2, q=1, \alpha_{1}=-n, \alpha_{2}=n+1, \rho_{1}=m+1$ and replace $z$ by $2 /(z-1)$; then

$$
(n+m+1) T_{n+1}^{-m}(z)-(2 n+1) z T_{n}^{-m}(z)+(n-m) T_{n-1}^{-m}(z)=0 .
$$

Next, in (7) put $p=2, q=1, \alpha_{1}=\frac{1}{2}(n+m), \alpha_{2}=\frac{1}{2}(n+m+1), \rho_{1}=n+\frac{1}{2}$, and replace $z$ by $-z^{2} ;$ then, since

$$
\begin{array}{r}
Q_{n}^{m}(z)=2^{m-1}\left(z^{2}-1\right)^{1 m} z^{-n-m-1} E\left(\frac{1}{2} n+\frac{1}{2} m+\frac{1}{2}, \frac{1}{2} n+\frac{1}{2} m+1: n+\frac{3}{2}:-z^{2}\right), \\
Q_{n-1}^{m}(z)-(2 n+1) \sqrt{ }\left(z^{2}-1\right) Q_{n}^{m-1}(z)-Q_{n+1}^{m}(z)=0 . \ldots \ldots \ldots \ldots
\end{array}
$$

From (12),

$$
\frac{d}{d z} E\left(: n+1: 4 / z^{2}\right)=-\frac{1}{2} z E\left(: n+2: 4 / z^{2}\right)
$$

and therefore, from (13),

Hence, from (14),

$$
J_{n}^{\prime}(z)-n z^{-1} J_{n}(z)=-J_{n+1}(z)
$$

The other derivatives can be derived in much the same way. For instance, for $K_{n}(z)$, apply (9) to the expression on the right of (12), and then use formula (15).

\section{REFERENCES}

1. Gauss, C. F., Werke III (1876), 130, 133.

2. MacRobert, T. M., Proofs of some formulae for the hypergeometric function, Phil. Mag. (7) 16 (1933), 440, 441, 442.

\section{The UNTVERSITY}

\section{Glasgow}

